Proceedings of the XXXVIII International School and Conference on the Physics of Semiconductors "Jaszowiec" 2009

\title{
Cross-Correlations and Charge Pumping in Shot Noise of Coupled Quantum Dot System
}

\author{
G. MichaŁeK And B.R. BuŁKA \\ Institute of Molecular Physics, Polish Academy of Sciences, M. Smoluchowskiego 17, 60-179 Poznań, Poland

\begin{abstract}
We analyze current auto- and cross-correlations in the system of two capacitively coupled large quantum dots. Our attention is focused on the strong inter-dot coupling when an electron transfer through one of the quantum dots induces charge pumping through the second quantum dot. We show individual contributions to shot noise from various tunneling processes in the charge space.
\end{abstract}

PACS numbers: 73.23.Hk, 73.63.Kv, 73.50.Td

\section{Introduction}

The shot noise analysis gives information about current correlations in mesoscopic devices. In general, the shot noise is reduced below a Poissonian value $S_{\mathrm{P}}=2 e I$ ( $I$ - an average current, $e-$ an electron charge) because of the Pauli exclusion principle of scattered particles. The reduction appears e.g. in quantum point contacts, metallic diffusive wires, chaotic cavities and single electron transistors (SETs) [1]. Electronic correlations can enhance shot noise above the Poissonian value, e.g. in resonant tunneling diodes and ferromagnetic SETs [1]. Recently, development of novel technologies allow to study not only auto-correlations on the same branch of the circuit but also cross-correlations between different branches of the multi-terminal devices [2]. In fermionic systems, in the absence of interactions, the Pauli exclusion principle can lead to antibunching which can be manifested in the negative cross-correlations. The positive cross-correlations in the fermionic systems are a characteristic signature of electron-electron interactions $[1,3]$.

In recent experiment McClure et al. [2] investigated gate-controlled sign reversal of noise cross-correlation in a simple four-terminal device with capacitively coupled quantum dots. The experiment was performed in the Coulomb blockade regime for small bias voltages and close to the border lines separating different Coulomb blockade regions. Our studies are more general, focused on dynamical aspects of current correlations in the system of two strongly coupled QDs for large bias voltages. In particular, we show that for a large enhancement of the auto-correlations above the Poissonian value is responsible a dynamical Coulomb blockade effect while negative cross-correlations are due to the strong potential fluctuations.

\section{Description of the double QD system}

A four-terminal device which consists of two quantum dots (QDs) coupled with external electrodes is considered, see inset to Fig. 1. We assume a strong electrostatic coupling between QDs and neglect tunneling between them. Electrical currents in the steady state are calculated by means of the method developed for a single QD with a continuous electronic density of states (DOS) [4], so

$$
\begin{aligned}
& I_{i \alpha}=-e(-1)^{\delta_{\mathrm{R} \alpha}} \sum_{n_{\mathrm{t}}, n_{\mathrm{b}}}\left[\Gamma_{i \alpha}^{+}\left(n_{\mathrm{t}}, n_{\mathrm{b}}\right)-\Gamma_{i \alpha}^{-}\left(n_{\mathrm{t}}, n_{\mathrm{b}}\right)\right] \\
& \quad \times p_{0}\left(n_{\mathrm{t}}, n_{\mathrm{b}}\right),
\end{aligned}
$$

where single electron tunneling rates through the junctions $i \alpha(i=\{\mathrm{t}, \mathrm{b}\}, \alpha=\{\mathrm{L}, \mathrm{R}\})$ are

$$
\begin{aligned}
& \Gamma_{i \alpha}^{ \pm}\left(n_{\mathrm{t}}, n_{\mathrm{b}}\right) \\
& \quad=\frac{1}{e^{2} R_{i \alpha}} \frac{\Delta F_{i \alpha}^{ \pm}\left(n_{\mathrm{t}}, n_{\mathrm{b}}\right)}{1-\exp \left[-\Delta F_{i \alpha}^{ \pm}\left(n_{\mathrm{t}}, n_{\mathrm{b}}\right) / k_{\mathrm{B}} T\right]} .
\end{aligned}
$$

$\Delta F_{i \alpha}^{ \pm}\left(n_{\mathrm{t}}, n_{\mathrm{b}}\right)$ describes a change of a free energy $F$ when one electron is added — " + " (extracted - "-") to (from) one of the QDs, $R_{i \alpha}$ is resistance of the junction $i \alpha, T-$ temperature and $e<0$ is an electron charge. Probabilities $p_{0}\left(n_{\mathrm{t}}, n_{\mathrm{b}}\right)$ to find the system in the state $\left(n_{\mathrm{t}}, n_{\mathrm{b}}\right)$ can be calculated from master equation $\mathrm{d} \widehat{\boldsymbol{p}} / \mathrm{d} t=\widehat{\boldsymbol{m}} \cdot \widehat{\boldsymbol{p}}$ with a left hand side equal to zero. In tunnel matrix $\widehat{\boldsymbol{m}}$ there are included only single-electron tunneling processes in the charge space $\left(n_{\mathrm{t}}, n_{\mathrm{b}}\right)$, while higher order processes are neglected.

The fluctuations in the system are analyzed within a generation-recombination approach [5] for multi-electron channels by a generalization of the method developed for spinless electrons in a SET [6]. According to this procedure the Fourier transform of the correlation function between quantities $X$ and $Y$ is

$$
\begin{aligned}
& S_{X Y}(\omega)=2 \sum_{n_{\mathrm{t}}, n_{\mathrm{b}}, n_{\mathrm{t}}^{\prime}, n_{\mathrm{b}}^{\prime}} \\
& \quad\left[X\left(n_{\mathrm{t}}^{\prime}, n_{\mathrm{b}}^{\prime}\right) G_{n_{\mathrm{t}}^{\prime}, n_{\mathrm{b}}^{\prime} ; n_{\mathrm{t}}, n_{\mathrm{b}}}(\omega) Y\left(n_{\mathrm{t}}, n_{\mathrm{b}}\right) p_{0}\left(n_{\mathrm{t}}, n_{\mathrm{b}}\right)\right. \\
& \left.\quad+Y\left(n_{\mathrm{t}}^{\prime}, n_{\mathrm{b}}^{\prime}\right) G_{n_{\mathrm{t}}^{\prime}, n_{\mathrm{b}}^{\prime} ; n_{\mathrm{t}}, n_{\mathrm{b}}}^{*}(\omega) X\left(n_{\mathrm{t}}, n_{\mathrm{b}}\right) p_{0}\left(n_{\mathrm{t}}, n_{\mathrm{b}}\right)\right],
\end{aligned}
$$

where $\widehat{\boldsymbol{G}}(\omega) \equiv 1 /[\mathrm{i} \omega \widehat{1}-\widehat{\boldsymbol{m}}]$. In the steady state the autoand cross-correlation functions between currents $I_{i \alpha}$ and $I_{j \beta}$ can be written as 

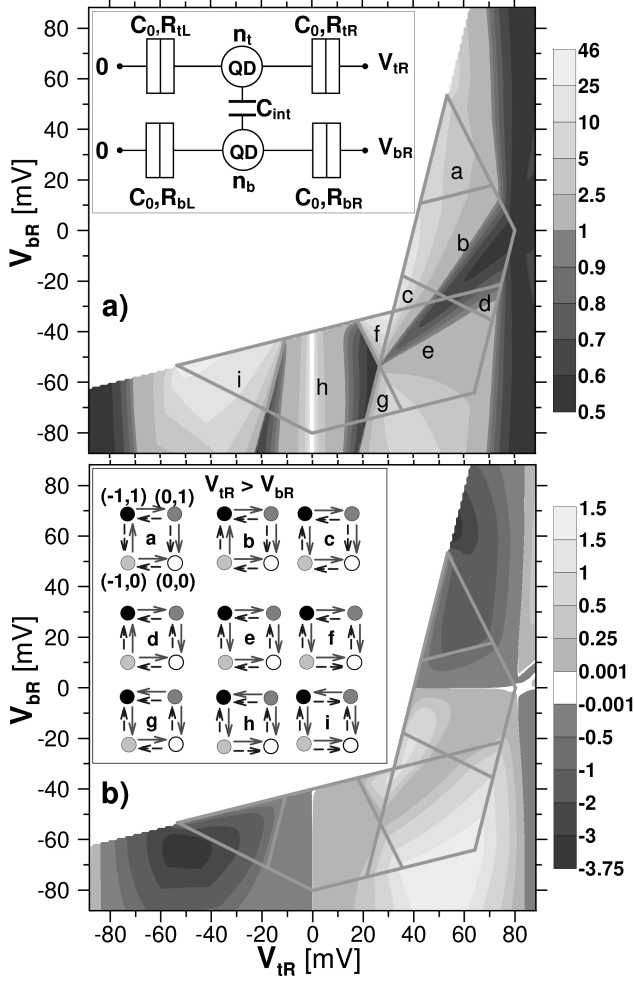

Fig. 1. (a) Map of the Fano factor $F_{\mathrm{tL}}=$ $S_{\mathrm{tL}, \mathrm{tL}}(0) / 2 e I_{\mathrm{tL}}$. (b) Map of the cross-correlation $S_{\mathrm{tL}, \mathrm{bL}}\left(10^{-28} \mathrm{~A}^{2} / \mathrm{Hz}\right)$. The results were performed for the $C_{\mathrm{int}}=2 \mathrm{aF}, C_{0}=1 \mathrm{aF}, R_{\mathrm{tL}}=R_{\mathrm{tR}}=1 \mathrm{M} \Omega$, $R_{\mathrm{bL}}=R_{\mathrm{bR}}=50 \mathrm{M} \Omega$ and $T=0 \mathrm{~K}$.

$$
S_{i \alpha, j \beta}(\omega)=\delta_{i j} \delta_{\alpha \beta} S_{i \alpha}^{\mathrm{Sch}}+S_{i \alpha, j \beta}^{\mathrm{c}}(\omega),
$$

where $S_{i \alpha}^{\text {Sch }}$ is a frequency independent Schottky part and $S_{i \alpha, j \beta}^{\mathrm{c}}(\omega)$ is frequency dependent [6].

\section{Results and conclusions}

Maps in Figs. 1a and $\mathrm{b}$ show the Fano factor $F_{\mathrm{tL}}=$ $S_{\mathrm{tL}, \mathrm{LL}}(\omega=0) / 2 e I_{\mathrm{tL}}$ in the top channel and cross-correlation function $S_{\mathrm{tL}, \mathrm{bL}}$ between the top and the bottom channels in the voltage range $V_{\mathrm{tR}}>V_{\mathrm{bR}}$ (results for voltages $V_{\mathrm{tR}}<V_{\mathrm{bR}}$ can be obtained by axial symmetry relative to $V_{\mathrm{tR}}=V_{\mathrm{bR}}$ line). The regions $\{a, \ldots, i\}$ correspond to tunnelings between four charge states $(0,0)$, $(-1,0),(-1,1),(0,1)$. In the inset of Fig. 1b we show all relevant tunneling processes through the left (solid arrows) and the right (dashed arrows) junctions contributing to transport in each region. Thick lines denote threshold voltages for (de-)activation of different $\Gamma_{i \alpha}^{ \pm}\left(n_{\mathrm{t}}, n_{\mathrm{b}}\right)$ tunneling rates. Let us start our analysis from region b. For small $V_{\mathrm{tR}}$ voltages $\left(V_{\mathrm{tR}}>V_{\mathrm{th}}\right)$ the auto-correlations are super-Poissonian, but one can see also a dark area (with $F_{\mathrm{tL}}<1$ ) inside this region for larger $V_{\mathrm{tR}}$ voltages. It indicates that there are various competing tunneling processes and their role changes with the bias voltage. The cross-correlation function $S_{\mathrm{tL}, \mathrm{bL}}$ is presented in Fig. 1b. The cross-correlations are negative when currents flow through the system in the same direction (i.e. when $I_{\mathrm{tL}} I_{\mathrm{bL}}>0$ ) and positive when $I_{\mathrm{tL}} I_{\mathrm{bL}}<0$. This dependence indicates on anti-bunching, but its origin is not fermionic as it was recently presented for electrons scattered in nanostructures [1]. Here, we have strong potential fluctuations which result in negative cross-correlations.

The Fano factor for the bottom QD $\left(F_{\mathrm{bL}}\right)$ is in general sub-Poissonian besides the narrow stripe around $V_{\mathrm{bR}}=0$ in the region b. In Fig. 2 we present the $V_{\mathrm{tR}}$ dependence of the main contribution to the current noise $S_{\mathrm{bL}}$ in the region $\mathrm{b}$ for $V_{\mathrm{bR}}=0 \mathrm{mV}$. One can see that the Schottky noise $S_{\mathrm{bL}}^{\mathrm{Sch}}$ is proportional to the tunneling current flowing through the top QD $\left(I_{\mathrm{tL}}\right)$, so the shot noise in the bottom QD is due to the charge fluctuations in the top QD. This charge pumping effect occurs when addition/extraction of one electron into/out the top QDs leads to pushing an electron out/into the bottom QDs.

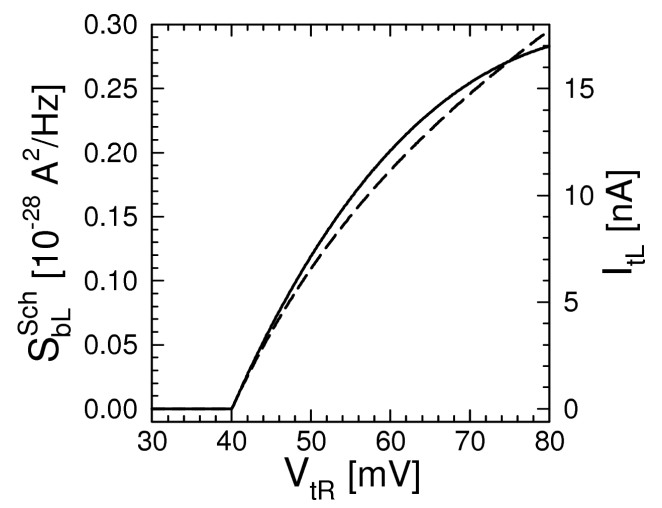

Fig. 2. The Schottky component $S_{\mathrm{bL}}^{\mathrm{Sch}}$ (solid line) and current $I_{\mathrm{tL}}$ (short-dashed line) for $V_{\mathrm{bR}}=0 \mathrm{mV}$ and $R_{\mathrm{bL}}=R_{\mathrm{bR}}=50 \mathrm{M} \Omega$. The other parameters are the same as in Fig. 1.

In order to find processes responsible for the behavior of shot noise presented in Fig. 1 we have calculated several cross-sections through the maps in Fig. 1. For example in Fig. 3a we show the cross-sections of the $F_{\mathrm{tL}}$ (solid line) and its components $\left(F_{\mathrm{tL}}^{\mathrm{Sch}}\right.$ - dash-dot line, $F_{\mathrm{tL}}^{\mathrm{c}}$ - short dash line) through the regions e, $\mathrm{f}, \mathrm{h}$ for $V_{\mathrm{bR}}=-50 \mathrm{mV}$. At small $V_{\mathrm{tR}}$ one can see a strong enhancement of the $F_{\mathrm{tL}}$ due to the Schottky term $S_{\mathrm{tL}}^{\mathrm{Sch}}$. For larger $V_{\mathrm{tR}}$ voltages a role of the contribution $S_{\mathrm{tL}}^{\mathrm{Sch}}$ decreases while the dynamical part $S_{\mathrm{tL}}^{\mathrm{c}}$ is negative. This leads to the reduction of the Fano factor below Poissonian value close to the border between regions $h$ and $f$. The super-Poissonian Fano factor appears only when $S_{\mathrm{tL}}^{\mathrm{c}}$ becomes positive. In order to understand a role of various tunneling processes in the shot noise $S_{\mathrm{tL}}$, we analyze all components, which contribute to the $S_{\mathrm{tL}}^{\mathrm{c}}$. In Fig. $3 \mathrm{~b}$ we present relevant components of the $S_{\mathrm{tL}}^{\mathrm{c}}$. One can see that in region $\mathrm{f}$ auto-correlation processes (i.e. processes proportional to $\left(\Gamma_{i \alpha}^{ \pm}\right)^{2}$ - curves with circles and squares) dominate over cross-correlation processes (i.e. processes proportional to $\Gamma_{i \alpha}^{ \pm} \Gamma_{j \beta}^{ \pm}$- curve with triangles) which 


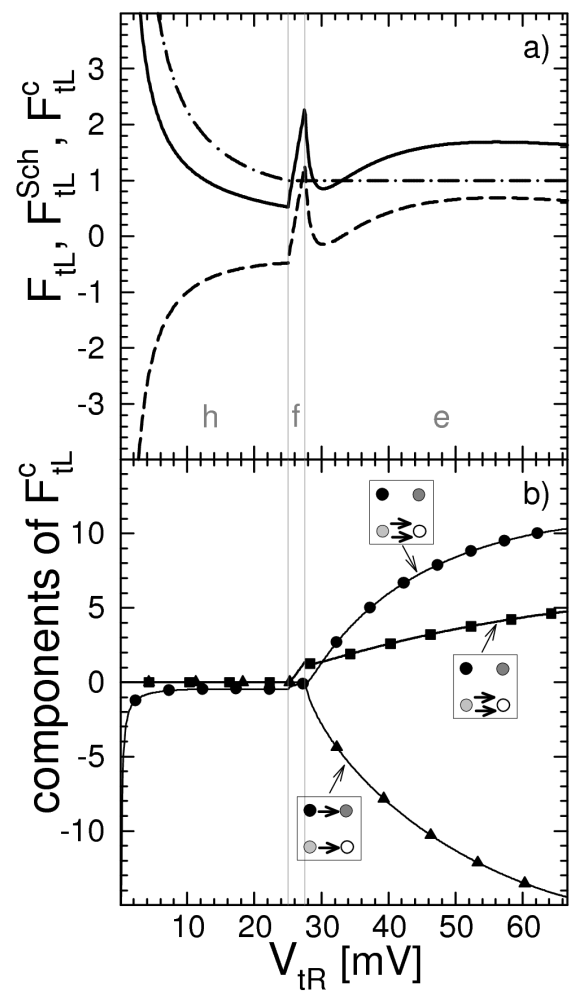

Fig. 3. (a) Total Fano factor $F_{\mathrm{tL}}$ (solid line), the Schottky term $F_{\mathrm{tL}}^{\mathrm{Sch}}$ (dash-dot line) and the dynamical contribution $F_{\mathrm{tL}}^{\mathrm{c}}$ (short dash line); and (b) relevant contributions to $F_{\mathrm{tL}}^{\mathrm{c}}$ plotted at $V_{\mathrm{bR}}=-50 \mathrm{mV}$. The other parameters are the same as in Fig. 1. lead to an enhancement of $F_{\mathrm{tL}}$ above Poissonian value. Furthermore, with increasing $V_{\mathrm{tR}}$, cross-correlations processes always decrease. The competition between positive auto- and negative cross-correlation processes can both enhance or reduce $S_{\mathrm{tL}}^{\mathrm{c}}$ leading to an enhancement or reduction of the $F_{\mathrm{tL}}$. These tunneling processes are responsible for non-monotonous behavior of the auto- and the cross-correlation functions.

\section{Acknowledgments}

The work was supported as part of the European Science Foundation EUROCORES Programme FoNE by funds from the Ministry of Science and Higher Education for the period 2006-2009.

\section{References}

[1] Ya.M. Blanter, M. Büttiker, Phys. Rep. 336, 1 (2000).

[2] D.T. McClure, L. DiCarlo, Y. Zhang, H.-A. Engel, C.M. Marcus, M.P. Hanson, A.C. Gossard, Phys. Rev. Lett. 98, 056801 (2007).

[3] B.R. Bułka, Phys. Rev. B 77, 165401 (2008) and references therein.

[4] G. Schön, Quantum Transport and Dissipation, Wiley-VCH Verlag, New York 1998, 149.

[5] K.M. van Vliet, J.R. Fassett, Fluctuation Phenomena in Solids, Academic Press, New York 1965, 267.

[6] A.N. Korotkov, Phys. Rev. B 49, 10381 (1994). 